\title{
Graphene Oxide Nanoparticles in the Interstellar Medium
}

\author{
P. J. Sarre, ${ }^{1 \star}$ \\ ${ }^{1}$ School of Chemistry, The University of Nottingham, University Park, Nottingham NG7 2RD, United Kingdom
}

Accepted XXX. Received YYY; in original form ZZZ

\begin{abstract}
Dust particles play a major role in the formation, evolution and chemistry of interstellar clouds, stars and planetary systems. Commonly identified forms include amorphous and crystalline carbon-rich particles and silicates. Also present in many astrophysical environments are polycyclic aromatic hydrocarbons (PAHs), detected through their infrared emission, and which are essentially small flakes of graphene. Astronomical observations over the past four decades have revealed a widespread unassigned 'Extended Red Emission' (ERE) feature which is attributed to luminescence of dust grains. Numerous potential carriers for ERE have been proposed but none has gained general acceptance. In this Letter it is shown that there is a strong similarity between laboratory optical emission spectra of graphene oxide and ERE, leading to this proposal that emission from graphene oxide nanoparticles is the origin of ERE and that these are a significant component of interstellar dust. The proposal is supported by infrared emission features detected by the Infrared Space Observatory (ISO) and the Spitzer Space Telescope.
\end{abstract}

Key words: astrochemistry - dust, extinction - methods: laboratory - stars: individual (Red Rectangle, HD 44179) - techniques: spectroscopic

\section{INTRODUCTION}

\subsection{Background}

Extended Red Emission (ERE) is a broad emission feature noted over forty years ago by Cohen et al. (1975) in a spectrophotometric study of the Red Rectangle - a mixedchemistry object which comprises a binary star, HD 44179, an oxygen-rich circumstellar disk, and an extended biconical carbon-rich nebula from which ERE emanates. It has since been detected in a very wide range of Galactic and extragalactic sources (Witt 2014; Lai et al. 2017). These include reflection nebulae, planetary nebulae, novae, H iI regions, high-latitude galactic cirrus clouds, the diffuse galactic medium and external galaxies - see Witt \& Schild (1988); Witt \& Boroson (1990); Scott et al. (1994); Szomoru \& Guhathakurta (1998); Smith \& Witt (2002); Rhee et al. (2007); Berné et al. (2008); Witt et al. (2008). ERE varies in peak wavelength (600-850 nm) and width (60-120 nm) both within and between objects.

Numerous carriers for ERE have been proposed but none of these is widely accepted. Proposals and carrier models published up to 2017 are discussed in Lai et al. (2017); these include hydrogenated amorphous carbon (HAC), polycyclic aromatic hydrocarbon (PAH) molecules, quenched carbon composite (QCC), $\mathrm{C}_{60}$, carbon clusters, silicon and

^ E-mail: peter.sarre@nottingham.ac.uk silicate nanoparticles, biofluorescence, magnesium silicate, nanodiamonds, PAH di-cations, and dimer cations, with hydrogen recently added (Holmlid 2018). ERE-like luminescence has also been detected in interplanetary dust particles and carbonaceous chondrites (Allamandola et al. 1987; Wopenka 1988; Quirico et al. 2005).

In this Letter oxidised graphene nanoparticles, small graphene oxide (GO) grains, are proposed as the ERE carrier based on comparison of laboratory photoluminescence (PL) of graphene oxide with ERE, and similarity in the spatial distribution of ERE and IR emission from chemical groups expected to be present in astronomical GO.

\subsection{ERE Carrier Characteristics}

A wide range of molecules and materials give rise to luminescence at red/near-IR wavelengths and, given the wavelength variation and breadth of the ERE feature, assignment of the carrier on these data alone presents a challenge. However, detailed studies have produced some general constraints (Witt \& Vijh 2004; Witt 2014; Lai et al. 2017). ERE is observed from carbon-rich but not oxygen-rich nebulae (Furton \& Witt 1990; Furton \& Witt 1992), strongly suggesting that the element carbon is involved; ERE is also seen in mixed-chemistry carbon-oxygen sources such as the Red Rectangle. Characteristics to be satisfied by proposed carriers include: an ERE peak wavelength - width relationship and a lack of ERE when the exciting star has $\mathrm{T}_{\text {eff }}$ 


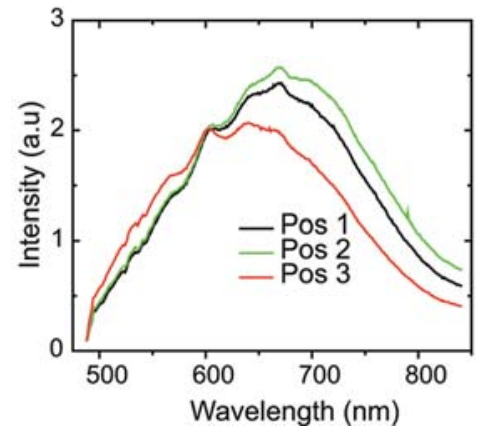

Figure 1. PL spectra of GO produced by an oxygen plasma treatment of graphene and excited by laser radiation at $473 \mathrm{~nm}$. Spectra for three irradiated positions (Pos 1,2 and 3) on the GO sample are shown. Reprinted with permission from Gokus et al. (2009). Copyright 2009 American Chemical Society.

$<10,000 \mathrm{~K}$ as described by Darbon et al. (1999), a lower limit on the estimated photon (not energy) conversion efficiency of about $10 \%$ for excitation in the visible-to-far-UV range (Gordon et al. 1998), and a requirement for photons with wavelengths short of $118 \mathrm{~nm}$ for ERE excitation in NGC 7023 (Witt et al. 2006). It is likely significant that a ubiquitous feature of many ERE-emitting sources is aromatic infrared band (AIB) emission from PAHs which is also induced by UV/vis excitation - see reviews by Tielens (2008, 2013). However, the spatial distribution of ERE- and PAHemitting regions is not identical from which it is inferred that there is not a direct one-to-one chemical correspondence between ERE carriers and PAHs in general (Witt 2014). Lai et al. (2017) have discussed likely photophysical mechanisms giving rise to the ERE emission.

\section{GO NANOPARTICLES AND ERE}

Pristine graphene is a single-layer planar network of sixmembered carbon rings in an $\mathrm{sp}^{2}$ bonding framework. It does not possess an optical band gap, the first electronic transition lying in the far-ultraviolet arising from a $\pi^{*}-$ $\pi$ transition. However, exposure of graphene to an oxygen plasma leads to incorporation of oxygen, forming graphene oxide which does have an optical band gap. Gokus et al. (2009) have shown that laser irradiation of GO generated in this way results in photoluminescence (PL) in the visible spectral range (Figure 1) where spectra for three spatially separate positions on the GO sample are shown with peak wavelengths of 630-660 nm. The spectra for Pos 1 and 2 on the oxidised graphene surface are almost identical and for Pos 3 the PL was bleached intentionally by using intense laser irradiation (Gokus et al. 2009). The PL was assigned as due to CO-related localised electronic states at the oxidation sites (Gokus et al. 2009). In an alternative approach yielding broadly similar results, Luo et al. (2009a) started with assynthesised GO (Luo et al. 2009b) and explored laser-excited $\mathrm{PL}$ spectra of solid and aqueous GO as the samples were progressively reduced with hydrazine.

Figure 2 shows ERE in the Red Rectangle $6^{\prime \prime}$ and $10^{\prime \prime}$ South of the central star HD 44179 (Witt \& Boroson 1990). ERE exhibits variation in peak wavelength and width both within the Red Rectangle, and also between objects; this is

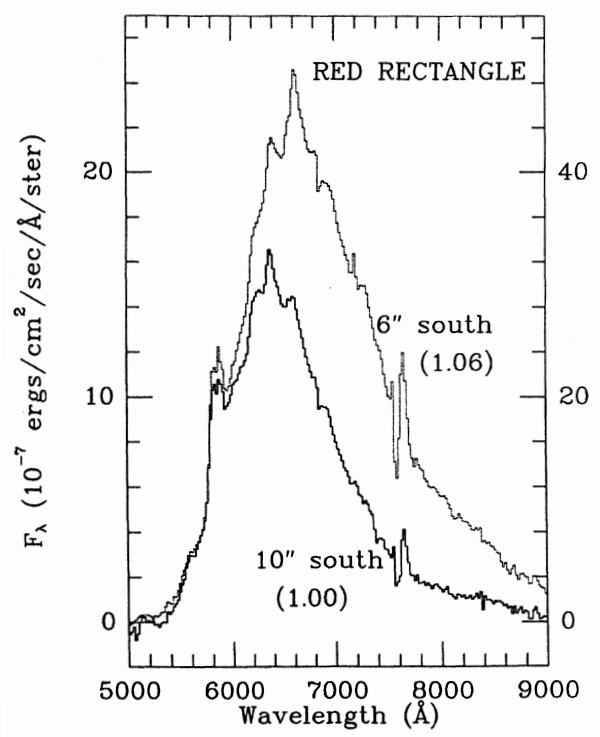

Figure 2. ERE in the Red Rectangle nebula $6^{\prime \prime}$ and $10^{\prime \prime}$ south of HD 44179. The figures in parentheses are the ratio of ERE to scattered light. Reproduced with permission from Witt \& Boroson (1990). The wavelength dispersion in figs 1 and 2 on the page is the same.

illustrated for a wider set of targets in fig. 7 of Ledoux et al. (2001). The peak wavelength and FWHM for the laboratory PL of GO (figure 1) and the Red Rectangle ERE (figure 2) at $6^{\prime \prime}$ are very similar, indicating that GO is a good candidate as the ERE carrier.

Laboratory samples of GO are generally produced by exfoliation of graphite oxide using methods such as the modified Hummers method in which four main oxygen-bearing sites are found - carbonyl $(>\mathrm{C}=\mathrm{O})$, epoxide $(-\mathrm{O}-)$, carboxylic acid $(-\mathrm{COOH})$ and hydroxyl $(-\mathrm{OH})$ groups, not all of which may be of astronomical relevance. Sites for these groups are illustrated schematically in figure 3 . The chemical structure of GO is a matter of ongoing discussion with a number of models proposed - for a summary see Dreyer et al. (2010); Zhao et al. (2015). In experiments by Cuong et al. (2011) thermal reduction at $700^{\circ} \mathrm{C}$ resulted in removal of many oxygen-containing groups illustrated in figure 3 , but with carbonyl groups being persistent. Li et al. (2012) have found that GO samples with high levels of carbonyl groups produce PL with the best spectral match to ERE. A second luminescence feature of GO is commonly seen near $400 \mathrm{~nm}$. This 'blue luminescence' is due to transitions involving confined $\mathrm{sp}^{2}$ bonded 'aromatic' regions (Chien et al. 2012; Yuan et al. 2019); the possible astrophysical relevance of this will be discussed elsewhere.

An important question, addressed in the following section, is whether there is evidence from astronomical observations for IR emission at wavelengths consistent with IR laboratory absorption spectra of GO due to these chemical groups, and whether the spatial distributions of ERE and oxygen-related IR emission features in astronomical objects are similar. 


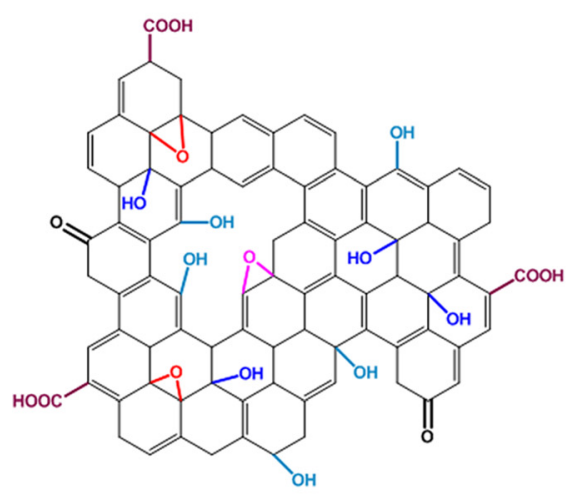

Figure 3. Schematic illustration of GO showing sites of oxygencontaining groups in as-produced (modified Hummers method) GO (Cuong et al. 2011). Hydrogen atoms (C-H) are omitted for clarity. The representation is based on theoretical work by Gao et al. (2010) and is reprinted from Cuong et al. (2011), with the permission of AIP Publishing.

\section{INFRARED SIGNATURES}

The carbonyl $\mathrm{C}=\mathrm{O}$ stretch transition is seen in laboratory absorption spectra of GO with a low level of oxidation (Krishnamoorthy et al. 2013). ISO spectra of the Red Rectangle have an emission feature at $6.0 \mu \mathrm{m}$ which is considered to be likely due to a $\mathrm{C}=\mathrm{O}$ stretch of a quinone-type PAH (Peeters et al. 2002). The ISO spectra are for a $14^{\prime \prime}$ x $20^{\prime \prime}$ exposure so direct comparison with the established bipolar-shaped ERE distribution is unfortunately not possible. Hsia et al. (2016) have collated data for 20 sources which exhibit the $6.0 \mu \mathrm{m}$ feature, eight of which are classified as mixed-chemistry objects. An alternative origin in olefinic double-bond functional groups was suggested (Hsia et al. 2016). However, taking gas-phase absorption data from Wallace (2019), carbonyl-containing molecules with one (1) or more $(2,3)$ aromatic rings - p-benzoquinone (1), 1,4 napthaquinone (2), anthrone (3) and anthraquinone (3) have expected peak emission wavelengths at $6.01,5.99,5.99$ and $5.98 \mu \mathrm{m}$, where the commonly invoked $15 \mathrm{~cm}^{-1}$ redward shift from absorption to astronomical emission has been invoked as discussed by Bauschlicher et al. (2009). From this it is deduced that attribution of the $6.0 \mu \mathrm{m}$ astrophysical emission band to the $\mathrm{C}=\mathrm{O}$ stretch of oxygenated PAHs is supported by laboratory data.

In $I S O$ spectra of a number of sources, Peeters et al. (2002) found that the $6.0 \mu \mathrm{m}$ band appears not to be correlated with the nearby carbon-carbon stretching band at $6.2 \mu \mathrm{m}$ and this is confirmed by Spitzer data for NGC 2023 where the G6.0 $0^{1}$ and 6.2 distributions do not match (Peeters et al. 2017). Within the SL FOV for the Southerly Spitzer observations of NGC 2023 (Peeters et al. 2017), the G6.0 feature peaks along the $\mathrm{S}$ ridge which differs greatly from the $6.2,7.7$ and $8.6 \mu \mathrm{m} \mathrm{AIBs,}$ and while it shares in part the distribution of the $11.2 \mu \mathrm{m}$ feature due to neutral PAHs, its distribution is closest to the $8 \mu \mathrm{m}$ 'bump' as presented by Peeters et al. (2017). The G6.0 distribution also has much in common with the $5-10 \mu \mathrm{m}$ and $10-15 \mu \mathrm{m}$ plots,

1 G refers to a Gaussian decomposition the $14.7 \mu \mathrm{m}$ continuum associated with Very Small Grains (VSGs), and with molecular hydrogen $\mathrm{S}(2)$ and $\mathrm{S}(3)$ emission as seen earlier in studies of 1-0 S(1) and other highly excited $\mathrm{H}_{2}$ lines which depend on UV excitation (McCartney et al. 1999). Taking the PAH and continuum Spitzer data across NGC 2023, Peeters et al. (2017) comment that the $6.0 \mu \mathrm{m}$ PAH emission 'seems to be somewhat unique', and a 'unique' spatial distribution is also found for the Northern map. Within the SL FOV, the S ridge is the region in which ERE emission is strongest (Witt \& Malin 1989; Pilleri 2010), indicating a link between $6.0 \mu \mathrm{m}$ emission and ERE. The SL northern map (Peeters et al. 2017) is an area of weak ERE emission (Witt \& Malin 1989) and a $6.0 \mu \mathrm{m}$ feature is found only in a very small region.

A second broader band centred at $8.0 \mu \mathrm{m}$ is seen in laboratory spectra of GO and is attributed to C-O-C epoxy group(s) (Krishnamoorthy et al. 2013). Although not classified as a formal PAH band in the decomposition of NGC 2023 spectra by Peeters et al. (2017) (their figure 2) a fairly broad feature in NGC 2023 is seen and termed the $8 \mu \mathrm{m}$ 'bump'. This 'bump' is not the same as the wide $8.0 \mu \mathrm{m}$ Spitzer IRAC filter which covers the $6.2,7.7$ and $8.6 \mu \mathrm{m}$ PAHs. Rather the 'bump' resembles a PAH-type band, though broader. Given that the spatial distribution of the 'bump' is very similar to the G6.0 band, a related origin for these two bands is suggested. In future work it would be of interest to compare the G6.0 and $8.0 \mu \mathrm{m}$ 'bump' intensities with ERE strength along sections in NGC 2023, NGC 7023 and other extended objects. Laboratory IR absorption by hydroxyl groups of GO has been reported near $2.9 \mu \mathrm{m}$ (Bagri et al. 2010) but it is much broader than other features and might be difficult to detect if present in the proposed astronomical GO; there is no evidence for such an emission feature in $I S O$ spectra of the Red Rectangle. In summary it is found that IR emission from the special sub-group of oxygenated PAH structures (GO) supports the attribution of ERE to GO.

Pilleri (2010) has shown that along sections taken orthogonal to ridges in NGC 2023 and NGC 7023, ERE peaks at the interface of neutral PAH and Very Small Grain (VSG) emission where VSGs with typical size of about 500 carbon atoms are being evaporated to form PAHs - as discussed by Cesarsky et al. (2000), Berné et al. (2007) and Croiset et al. (2016). This suggests that the GO particle size falls roughly in the same range as the well-studied PAHs. In the context of this proposal VSGs could include multilayer graphite oxide grains which split into individual GO layers on becoming separated by exposure to UV, shocks etc.

\section{FURTHER COMMENTS}

Laboratory studies show that luminescence of GO occurs when it is excited with visible light (Gokus et al. 2009). This might be considered inconsistent with the astrophysical constraint that UV irradiation is a prerequisite for ERE emission (Smith \& Witt 2002; Witt 2014). However, if the formation of GO in astrophysical environments results from decomposition of multi-layered graphite oxide under UV irradiation, the need for UV would be satisfied. A second possibility is that GO formation depends on creation of radical sites e.g. through photo-removal of $\mathrm{H}$ from PAH structures, 
and replacement by $\mathrm{O}$ to form carbonyl or epoxy groups. The principle of a two-step process has been invoked for other proposed ERE carriers (Witt et al. 2006; Rhee et al. 2007).

The attribution of ERE to GO can be tested by laboratory experiments on GO nanoparticles in which the degree of oxidation is controlled, the temperature of the sample varied and broad-band excitation is employed. In the Red Rectangle the peak wavelength is reported to change from $\sim 755 \mathrm{~nm}$ about $2^{\prime \prime}$ South of HD 44179 (Rouan et al. 1995; Ledoux et al. 2001) to $~ 670 \mathrm{~nm} 6^{\prime \prime}$ South and $645 \mathrm{~nm} \mathrm{10"}$ South (Witt \& Boroson 1990). While this might be due to a compositional change in the carrier, it is important to determine the effect of temperature and particle size on GO nanoparticle emission.

If confirmed, the presence of GO nanoparticles could have implications in other areas of astrochemistry. In the Red Rectangle a set of unidentified molecular emission features (Schmidt et al. 1980), sits atop the ERE feature, the carriers of which have been suggested to be decomposition products of the ERE-emitting material (Schmidt \& Witt 1991). Could oxygen-containing PAHs and related large carbonaceous molecules be the carriers of these Red Rectangle bands which are very strong near 5,800 $\AA$ and 6,600 $⿱$ ? These would be large organic 'dye' molecules in a chemical sense (Sarre 2006). An association of these emission bands with a subset of diffuse interstellar band carriers has been suggested (Sarre 1991; Fossey 1991; Sarre et al. 1995).

Other areas worthy of examination include (i) a known laboratory emission feature of GO near $400 \mathrm{~nm}$ which originates in $\mathrm{sp}^{2}$ graphitic islands and which might contribute to astronomical 'Blue Luminescence' (Vijh et al. 2005), (ii) any contribution of GO to, and variation in, the $2175 \AA$ interstellar absorption feature and (iii) the 'missing oxygen' problem. Catalytic reactions of atoms and molecules on GO surfaces could also be investigated.

\section{ACKNOWLEDGEMENT}

I thank the Leverhulme Trust for award of a Leverhulme Emeritus Fellowship.

\section{REFERENCES}

Allamandola L. J., Sandford S. A., Wopenka B., 1987, Science, 237,56

Bagri A., Mattevi C., Acik M., Chabal Y. J., Chhowalla M., Shenoy V. B., 2010, Nature Chemistry, 2, 581

Bauschlicher Charles W. J., Peeters E., Allamandola L. J., 2009, ApJ, 697, 311

Berné O., et al., 2007, A\&A, 469, 575

Berné O., Joblin C., Rapacioli M., Thomas J., Cuillandre J. C., Deville Y., 2008, A\&A, 479, L41

Cesarsky D., Lequeux J., Ryter C., Gérin M., 2000, A\&A, 354, L87

Chien C.-T., et al., 2012, Angewandte Chemie-International Edition, 51, 6662

Cohen M., et al., 1975, ApJ, 196, 179

Croiset B. A., Candian A., Berné O., Tielens A. G. G. M., 2016, A\&A, 590, A26

Cuong T. V., et al., 2011, App. Phys. Lett., 99, 041905

Darbon S., Perrin J.-M., Sivan J.-P., 1999, A\&A, 348, 990
Dreyer D. R., Park S., Bielawski C. W., Ruoff R. S., 2010, Chem. Soc. Rev., 39, 228

Fossey S. J., 1991, Nature, 353, 393

Furton D. G., Witt A. N., 1990, ApJ, 364, L45

Furton D. G., Witt A. N., 1992, ApJ, 386, 587

Gao X., Jang J., Nagase S., 2010, J. Phys. Chem C, 114, 832

Gokus T., et al., 2009, ACS nano, 3, 3963

Gordon K. D., Witt A. N., Friedmann B. C., 1998, ApJ, 498, 522

Holmlid L., 2018, ApJ, 866, 107

Hsia C.-H., Sadjadi S., Zhang Y., Kwok S., 2016, ApJ, 832, 213

Krishnamoorthy K., Veerapandian M., Yun K., Kim S.-J., 2013, Carbon, 53, 38

Lai T. S.-Y., Witt A. N., Crawford K., 2017, MNRAS, 469, 4933

Ledoux G., Guillois O., Huisken F., Kohn B., Porterat D., Reynaud C., 2001, A\&A, 377, 707

Li M., Cushing S. K., Zhou X., Guo S., Wu N., 2012, J. Mat. Chem., 22, 23374

Luo Z., Vora P. M., Mele E. J., Johnson A. T. C., Kikkawa J. M., 2009a, App. Phys. Lett., 94

Luo Z., Lu Y., Somers L. A., Johnson A. T. C., 2009b, JACS, 131,898

McCartney M. S. K., Brand P. W. J. L., Burton M. G., Chrysostomou A., 1999, MNRAS, 307, 315

Peeters E., Hony S., Van Kerckhoven C., Tielens A. G. G. M., Allamandola L. J., Hudgins D. M., Bauschlicher C. W., 2002, A\&A, 390, 1089

Peeters E., Bauschlicher C. W., Allamandola L. J., Tielens A. G. G. M., Ricca A., Wolfire M. G., 2017, ApJ, 836, 198

Pilleri P., 2010, PhD thesis, L'Université de Toulouse

Quirico E., Borg J., Raynal P., Montagnac G., d'Hendecourt L., 2005, Planetary and Space Science, 53, 1443

Rhee Y. M., Lee T. J., Gudipati M. S., Allamandola L. J., HeadGordon M., 2007, Proceedings of the National Academy of Science, 104, 5274

Rouan D., Lecoupanec P., Léger A., 1995, in Jeffery C., ed., Proc. 1st Franco-British Meeting on the Physics and Chemistry of the Interstellar Medium, Newsletter on Analysis of Astronomical Spectra. No. 22 in 1. p. 37

Sarre P. J., 1991, Nature, 351, 356

Sarre P. J., 2006, Journal of Molecular Spectroscopy, 238, 1

Sarre P. J., Miles J. R., Scarrott S. M., 1995, Science, 269, 674

Schmidt G. D., Witt A. N., 1991, ApJ, 383, 698

Schmidt G. D., Cohen M., Margon B., 1980, ApJ, 239, L133

Scott A. D., Evans A., Rawlings J. M. C., 1994, MNRAS, 269, L21

Smith T. L., Witt A. N., 2002, ApJ, 565, 304

Szomoru A., Guhathakurta P., 1998, ApJ, 494, L93

Tielens A. G. G. M., 2008, ARA\&A, 46, 289

Tielens A. G. G. M., 2013, Reviews of Modern Physics, 85, 1021

Vijh U. P., Witt A. N., Gordon K. D., 2005, ApJ, 633, 262

Wallace W. E., 2019, in Linstrom P. J., Mallard W. G., eds, NIST Chemistry WebBook, NIST Standard Reference Database Number 69, https://doi.org/10.18434/T4D303. https ://doi. org/10.18434/T4D303

Witt A. N., 2014, in Cami J., Cox N. L. J., eds, IAU Symposium Vol. 297, The Diffuse Interstellar Bands. pp 173-179, doi:10.1017/S1743921313015810

Witt A. N., Boroson T. A., 1990, ApJ, 355, 182

Witt A. N., Malin D. F., 1989, ApJ, 347, 25

Witt A. N., Schild R. E., 1988, ApJ, 325, 837

Witt A. N., Vijh U. P., 2004, in Witt A. N., Clayton G. C., Draine B. T., eds, Astronomical Society of the Pacific Conference Series Vol. 309, Astrophysics of Dust. p. 115 (arXiv:astro$\mathrm{ph} / 0309674)$

Witt A. N., Gordon K. D., Vijh U. P., Sell P. H., Smith T. L., Xie R.-H., 2006, ApJ, 636, 303

Witt A. N., Mandel S., Sell P. H., Dixon T., Vijh U. P., 2008, ApJ, 679, 497 
Wopenka B., 1988, Earth and Planetary Science Letters, 88, 221

Yuan T., Meng T., He P., Shi Y., Li Y., Li X., Fan L., Yang S., 2019, J. Mat. Chem., 7, 6820

Zhao J., Liu L., Li F., 2015, Graphene Oxide, Physics and Applications. Springer

This paper has been typeset from a $\mathrm{T}_{\mathrm{E}} \mathrm{X} / \mathrm{LAT}_{\mathrm{E}} \mathrm{X}$ file prepared by the author. 